\title{
VARIABILIDADE ESPACIAL DA ESTABILIDADE DOS AGREGADOS E MATÉRIA ORGÂNICA DO SOLO EM TERRA PRETA ARQUEOLÓGICA SOB PASTAGEM
}

\author{
Marcelo Dayron Rodrigues Soares ${ }^{1 *}$, Milton César Costa Campos ${ }^{1}$, José Maurício da Cunha ${ }^{1}$, \\ Bruno Campos Mantovanelli ${ }^{2}$, Ivanildo Amorim de Oliveira ${ }^{3}$, Elilson Gomes de Brito Filho ${ }^{1}$, \\ Alan Ferreira Lima Leite ${ }^{1}$
}

${ }^{1}$ Universidade Federal do Amazonas, UFAM, Humaitá, AM-Brasil, CEP: 69.800-000.

${ }^{2}$ Universidade Federal de Santa Maria, UFSM, Santa Maria, RS-Brasil, CEP: 97105 - 900

${ }^{3}$ Instituto Federal de Educação, Ciência e Tecnologia do Pará, IFPA, Breves, PA-Brasil, CEP: 68.800-000

*Autor para correspondência: marcelo.dayron@gmail.com

Recebido em 24 de maio de 2017. Aceito em 19 de maio de 2018. Publicado em 20 de junho de 2018.

REsumo - A agregação do solo pode representar um parâmetro que relaciona o manejo à qualidade do solo para as terras pretas arqueológicas (TPA). O objetivo do trabalho foi estudar a variabilidade espacial dos agregados e matéria orgânica do solo em terra preta arqueológica sob pastagem. Os dados foram coletados em uma parcela amostral com as dimensões de 80 x $56 \mathrm{~m}$, com espaço entre os pontos de $08 \mathrm{~m}$, e total de 88 pontos amostrais. As amostras de solos foram coletadas nas profundidades 0,0-0,05, 0,05-0,10 e 0,10-0,20 m, para as avaliações: diâmetro médio geométrico (DMG), classe de agregados $>2,00 \mathrm{~mm}, 2-1 \mathrm{~mm}$, índice da estabilidade dos agregados (IEA) e matéria orgânica do solo (MO); após análise exploratória e geoestatística, os modelos foram ajustados aos semivariogramas para cada atributo. Os resultados mostraram que a porcentagem de agregados na classe $>2,00 \mathrm{~mm}$ apresentou valores acima de $80 \%$ indicando maior resistência à desagregação. Os altos teores de MO no solo influenciaram a formação e estabilização dos agregados do solo.

Palavras-chave: Estrutura do Solo; Agregaç̃o; Geoestatística.

SPATIAL VARIABILITY OF SOIL AGGREGATES AND SOIL ORGANIC MATTER IN ARCHAEOLOGICAL BLACK EARTH UNDER PASTURE

AвSTRACт - The soil aggregation may represent a parameter that relates handling to soil quality for archaeological black earth (TPA). The objective was to study the spatial variability of the aggregates and soil organic matter in archaeological black earth under pasture. The data were collected in a sample plot with dimensions of 80 x $56 \mathrm{~m}$, with space between the points of $08 \mathrm{~m}$, and total of 88 sampling points. Soil samples were collected at depths $0.0-0.05,0.05-0.10$ and 0.10 to $20 \mathrm{~m}$, for evaluations: geometric mean diameter (GMD), aggregate class $>2,00 \mathrm{~mm}, 2-1 \mathrm{~mm}$, the aggregate stability index (ESI) and soil organic matter (SOM); after exploratory and geostatistical analysis, the models were fitted to semivariograms for each attribute. The results showed that the percentage of aggregates in the class $>2.00 \mathrm{~mm}$ had values above $80 \%$, indicating greater resistance to breakdown. The high SOM content in the soil influenced the formation and stabilization of soil aggregates.

Keywords: Soll Structure; AgGregAtion; Geostatistics.

VARIABILIDAD ESPACIAL DE LA ESTABILIDAD DE AGREGADOS Y MATERIA ORGÁNICA DEL SUELO EN TIERRA NEGRA ARQUEOLÓGICA BAJO PASTOREO

RESUMEN - La agregación del suelo puede representar un parámetro que relaciona el manejo con la calidad del suelo para la tierra negra arqueológica (TNA). El objetivo de este estudio fue investigar la variabilidad espacial de los agregados 
y de la materia orgánica del suelo en tierra negra arqueológica bajo pastoreo. Se recolectaron los datos en una parcela muestral con dimensiones de 80 × 56 m, con 8 m de espacio entre los puntos, y un total de 88 puntos muestrales. Las muestras de suelo se recolectaron en las profundidades de $0,0-0,05,0,05-0,10$ y $0,10-20 \mathrm{~m}$, para los análisis de diámetro medio geométrico (DMG), clase de agregados $>2,00 \mathrm{~mm}, 2-1 \mathrm{~mm}$, índice de estabilidad de los agregados (IEA) y materia orgánica del suelo (MO). Después de los análisis exploratorio y geoestadístico, los modelos se ajustaron a los semivariogramas para cada atributo. Los resultados mostraron porcentajes por encima del $80 \%$ para los agregados en la clase $>2,00 \mathrm{~mm}$, indicando una mayor resistencia a la desagregación. Los altos contenidos de MO en el suelo influenciaron la formación y estabilización de sus agregados.

Palabras clave: Estructura del suelo; Agregación; Geoestadística.

\section{INTRODUÇão}

As áreas de terras pretas arqueológicas (TPAs), apresentam elevada fertilidade quando comparado aos solos circunvizinhos que predominam na Amazônia, por esse motivo, são muito utilizadas para o cultivo de subsistência dos ribeirinhos. Por outro lado, as ações de manejo incorreto das pastagens nesses ambientes, resultam em degradação e, aceleram o processo de erosão dos solos. Segundo relatos de Araújo et al. (2011), a principal causa do desmatamento na região Amazônica é a expansão da pecuária bovina que tem como princípio a remoção e conversão da floresta em áreas de pastagem.

De acordo com Neves et al. (2006), a agregação é uma das propriedades que podem ser utilizadas para avaliar a qualidade do solo, uma vez que a manutenção de sua estrutura facilita a aeração e a infiltração de água e reduz a erodibilidade. Corrêa et al. (2009) relata que o processo de agregação envolve um conjunto de elementos, entre eles, a matéria orgânica, que atua como agente cimentante unindo as partículas do solo.

A caracterização e agregação do solo têm sido alvo de muitos estudos em TPAs, cujo resultados tem se mostrado importantes para região. Ao examinar os atributos físicos do solo em áreas sob diferentes sistemas de usos na região de Manicoré, AM, Soares et al. (2016) encontrou os menores valores de DMG para o cultivo de milho em solos antropogênicos. Por outro lado, Oliveira et al. (2015) ao avaliar a distribuição de tamanho dos agregados e outros atributos do solo em seis manejos e verificaram maior diâmetro de agregados do solo em TPA. Aquino et al. (2014), em pesquisa com variabilidade espacial em solo antropogênico e não antropogênico, apontaram elevada estabilidade para os solos antropogênicos. Neste contexto, pesquisas com a espacialização da estabilidade dos agregados do solo submetidos a manejos diferenciados têm sido realizado, os quais destacam-se: variação espacial dos agregados em sob diferentes usos (Gomes et al. 2017), dependência espacial da estabilidade dos agregados em terra preta de índio sob cultivo de cacau (Silva et al. 2016), agregados e carbono orgânico do solo em campo natural e floresta Alho et al. (2014),

De modo geral, a elevada estabilidade dos agregados em TPAs, é atribuída ao elevado teor de matéria orgânica, característico deste solo. Todavia, os trabalhos que se concentram na avaliação da variação espacial em áreas de TPAs, foram realizados recentemente, e pesquisas voltadas para esses solos, são subsídios para pesquisas futuras.

Diante do exposto, o objetivo deste estudo foi verificar a variabilidade espacial dos agregados e matéria orgânica do solo em terra preta arqueológica sob pastagem na região de Manicoré, AM. 


\section{MATERIAL E MÉTODOS}

A área de estudo está localizada ao sul do Estado do Amazonas, às margens da BR 230, rodovia Transamazônica, no município de Manicoré, sob as coordenadas geográficas de $73^{\circ}$ 0’22" de latitude sul e $63^{\circ}$ 01'15” de longitude oeste com altitude em torno de 83 m (Figura 1), sob Latossolo Amarelo Ditrófico psamítico, textura arenosa, horizonte A antrópico, apresentando teores médios de areia, silte e argila de 710, 221 e $65 \mathrm{~g} \mathrm{~kg}^{-1}$, respectivamente.

O clima da região segundo a classificação de Köppen, é do tipo tropical chuvoso, apresentando um período seco de pequena duração (Am), temperaturas variando entre 25 e $27{ }^{\circ} \mathrm{C}$ e precipitação média anual de 2.500 mm (BRASIL, 1978). O material de origem é proveniente da Formação Içá, caracterizada com produto de sedimentação pleistocênica, coberta por depósitos eólicos. O relevo é constituído por platôs com superfícies planas, sendo a zona de borda marcada por colinas e cristas alinhadas e localmente escarpadas (CPRM, 2001). A vegetação primária da região é Floresta Tropical Densa.

A área de TPA é composta por pastagem implantada há sete anos com a espécie Brachiaria brizanta, em sistema de pastejo extensivo e a capacidade de suporte de animais de um (1) unidade animal por hectare. Foi construído uma grade amostral contendo 88 pontos, espaçados em $08 \mathrm{~m}$, nas dimensões de 80 x $56 \mathrm{~m}$. No cruzamento das linhas da grade, o solo foi amostrado nas profundidades: 0-0,05, 0,05-0,10, 0,10-0,20m.

Figura 1 - Mapa de localização da área de estudo.

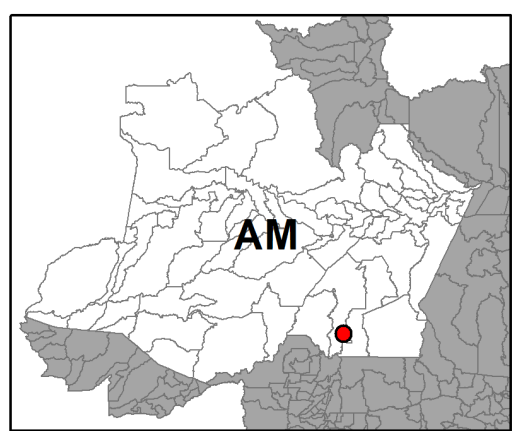

Legenda

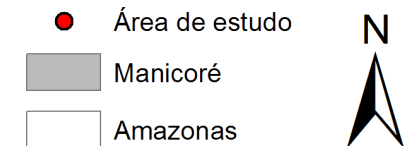

0

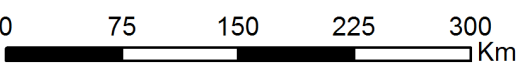

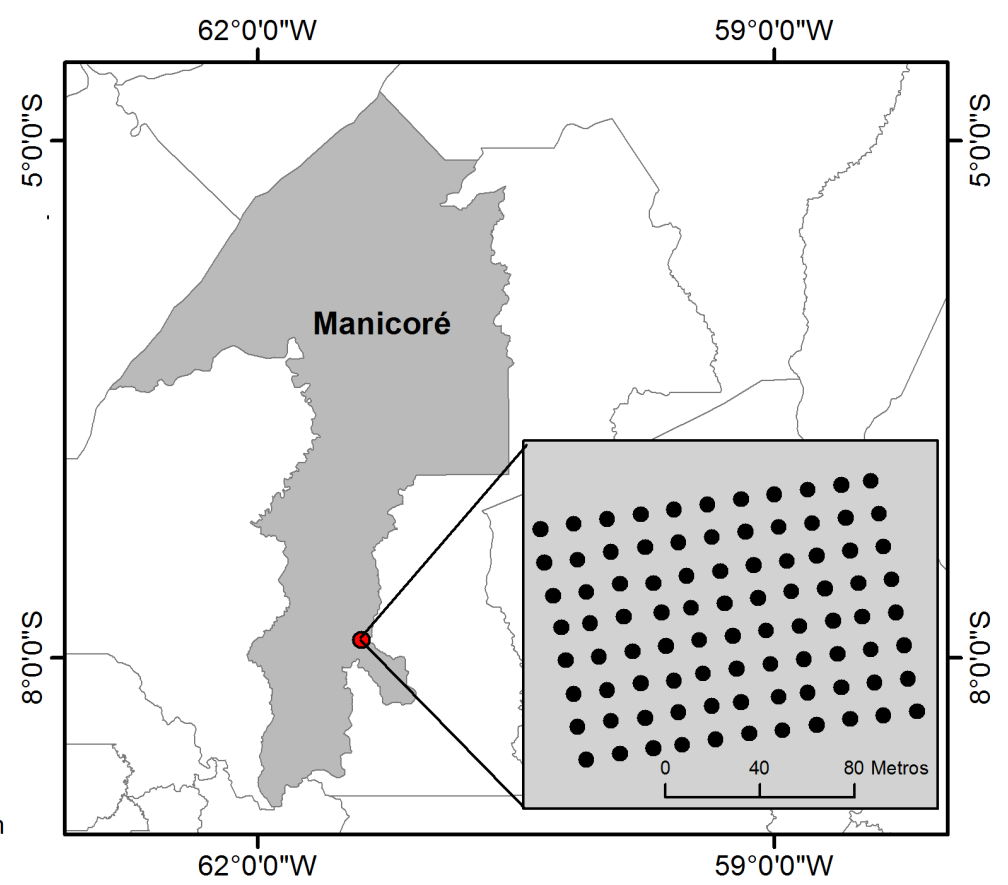

$62^{\circ} 0^{\prime} \mathrm{O}^{\prime \prime} \mathrm{W}$

$59^{\circ} 0^{\prime} \mathrm{O}^{\prime \prime} \mathrm{W}$

$\mathrm{Na}$ avaliação da estabilidade de agregados, amostras de solo foram coletadas amostras indeformadas. Posteriormente, as amostras foram pré-selecionadas, secas ao ar, acondicionadas e agitadas em jogo de peneiras sobrepostas, tendo as peneiras superior e inferior de $9,52 \mathrm{~mm}$ e $4,76 \mathrm{~mm}$, respectivamente. Em seguida, $20 \mathrm{~g}$ de cada amostra retida na peneira de $4,76 \mathrm{~mm}$, foi empregada na análise da estabilidade de agregados via úmida, colocadas sobre um jogo de peneiras com tamisagem de 2,0 mm;1,0 mm;0,5 mm;0,25 mm;0,105 $\mathrm{mm}$; e < $0,105 \mathrm{~mm}$ e submetendo-os a oscilações verticais. A separação e estabilidade dos agregados foram determinadas 
conforme Kemper \& Chepil (1965). Os resultados foram expressos em porcentual dos agregados retidos nas peneiras $>2 \mathrm{~mm}, 2-1 \mathrm{~mm}$, diâmetro médio geométrico (DMG), e o índice de estabilidade de agregados (IEA), por meio da equação IEA $=\left(\mathrm{M}_{\mathrm{T}}-\mathrm{M}_{<0,105} / \mathrm{M}_{\mathrm{T}}\right)$, em que MT é massa total de agregados descontado o teor de água (g) e $\mathrm{M}_{<0,105}$ é a massa de agregados da classe $<0,105 \mathrm{~mm}$ (g). A determinação da matéria orgânica foi realizada conforme Raij et al. (1987).

Para determinação da variabilidade espacial foi utilizada a análise geoestatística. O semivariograma experimental foi estimado pela equação (1).

$$
\begin{gathered}
\widehat{\Xi}(h)=\frac{1}{2 N(h)} \sum_{i=1}^{N(h)}\left[Z\left(x_{i}\right)-Z\left(x_{i}+h\right)\right]^{2} \\
Y
\end{gathered}
$$

Sendo: $\gamma(h)$ - valor da semivariância para uma distância $h ; \mathrm{N}(\mathrm{h})$ - número de pares envolvidos no cálculo da semivariância; $\mathrm{Z}\left(\mathrm{x}_{\mathrm{i}}\right)$ - valor do atributo $\mathrm{Z}$ na posição $\mathrm{x}_{\mathrm{i}} ; \mathrm{Z}\left(\mathrm{x}_{\mathrm{i}}+\mathrm{h}\right)$ - valor do atributo $\mathrm{Z}$ separado por uma distância $h$ da posição $x_{i}$.

Para a análise dos dados foi realizada, a estatística descritiva dos dados e à hipótese de normalidade dos dados pelo teste de Kolmogorov-Smirnov, a nível de 5\% de probabilidade, através do software Minitab. O coeficiente de variação $(\mathrm{CV})$ foi considerado baixo quando $\mathrm{CV}<12 \%$; médio para o intervalo $12-60 \%$; alto quando CV>60\%, (Warrick \& Nielsen 1980).

Selecionou-se o melhor modelo por meio da validação cruzada (VC), e o coeficiente de determinação $\left(\mathrm{r}^{2}\right)$ pelo software $\mathrm{GS}^{+}$. Portanto, na escolha entre diversos modelos o melhor é o que tiver maior valor de (VC). Para a confecção dos mapas espaciais utilizou-se o software Surfer versão 8.0.

A análise de dependência espacial (DE) foi realizada segundo Cambardella et al. (2014) que preconizam a proporção em porcentagem do efeito pepita $\left(C_{0}\right)$ em relação ao patamar $\left(C_{0}+C_{1}\right)$ e apresentam: dependência forte $<25 \%$; dependência moderada entre 25 e $75 \%$ e dependência fraca $>75 \%$.

\section{RESUltados E Discussão}

Os resultados referentes à estatística descritiva são apresentados na Tabela 1. O DMG, IEA, MO e as classes de agregados exibiram valores de média e mediana próximos, indicando distribuição normal. A maioria dos atributos apresentam valores positivos de curtose, os valores de assimetria e curtose ficaram próximos de zero, com exceção para IEA na camada 0-0,05 m.

Os resultados referentes ao teste Kolmogorov-Smirnov indicaram normalidade para $\mathrm{MO}$ em todas as camadas e para o DMG na camada superficial. Observou-se que os valores de DMG e MO foram elevados, a porcentagem de agregados na classe $>2,00 \mathrm{~mm}$, apresentou valores acima de $80 \%$ indicando maior resistência à desagregação. Para Oliveira et al. (2015), solos com TPA apresentam maior teor de MO, o que lhe confere maior fertilidade. De acordo com Hickman et al. (2011), o DMG é uma estimativa das classes de agregados de maior expressão.

O IEA mostrou-se adequado para avaliação da estabilidade dos agregados, seguindo o comportamento semelhante ao DMP. Vasconcelos et al. (2010) em estudos com estabilidade de agregados de um Latossolo Amarelo, afirmaram que o IEA e a percentagem de agregados maiores que 2,0 $\mathrm{mm}$, demonstram ser uma propriedade importante na avaliação da estabilidade de agregados. Neste sentido, Oliveira et al. (2013), 
trabalhando com estabilidade dos agregados sob diferentes usos comparando-os com mata, encontraram elevada estabilidade dos agregados em superfície.

É válido ressaltar que a formação de macroagregados é em função ação de manejos, especialmente em áreas de pastagens e podem não apresentar estabilidade. Segundo Luciano et al. (2010), valores elevados de DMG indicam solos com agregados estáveis, porém, pode ser resultado de compactação, configurando agregados de baixa qualidade. Neste aspecto, é válido ressaltar que o tamanho dos agregados é influenciado pelo uso e manejo do solo. No geral, os agregados de maior tamanho são formados por agentes cimentantes orgânico, principalmente pelas frações da matéria orgânica, especialmente exsudados orgânicos e material orgânico coloidal, podendo justificar a alta agregação deste estudo.

Em relação a estudos da estabilidade dos agregados do solo em TPAs, Campos et al. (2012), relatam que os solos de origem antrópica concentram grande quantidade de matéria orgânica derivada da queima parcial do carvão vegetal, deixando este solo mais solto e como consequência a predominância em formação de microagregados.

Os resultados evidenciam que o processo de estabilização dos agregados desse solo está associado ao teor de matéria orgânica. Na camada superficial, principalmente, à medida que o teor de MO diminui, há decréscimo na estabilidade dos agregados desse solo.

Tabela 1 - Estatística descritiva em relação ao diâmetro médio geométrico (DMG) e porcentagem dos agregados nas classes $>2 \mathrm{~mm}$ e na classe $2-1 \mathrm{~mm}$, índice de estabilidade de agregados (IEA) e matéria orgânica (MO) em diferentes profundidades.

\begin{tabular}{lccccc}
\hline Estatística & DMG $(\mathrm{mm})$ & $>2 \mathrm{~mm}(\%)$ & $2-1 \mathrm{~mm}(\%)$ & $\mathrm{IEA}(\%)$ & $\mathrm{MO}\left(\mathrm{g} \mathrm{dm}^{-3}\right)$ \\
\cline { 2 - 5 } Descritiva & \multicolumn{5}{c}{$0-0,05 \mathrm{~m}$} \\
\hline Média & 2,73 & 91,26 & 1,74 & 98,47 & 236,30 \\
Mediana & 2,74 & 92,4 & 1,40 & 98,89 & 200,68 \\
CV & 9,28 & 4,33 & 65,23 & 1,33 & 4,06 \\
Curtose & $-0,05$ & 1,51 & 0,22 & 4,67 & 1,05 \\
Assimetria & $-0,56$ & $-1,29$ & 1,09 & $-1,81$ & $-0,72$ \\
$\mathrm{~d}$ & $0,15 *$ & 0,01 & 0,01 & 0,01 & $0,15^{*}$ \\
\hline \multicolumn{5}{c}{$0,05-0,10 \mathrm{~m}$} \\
\hline Média & 2,76 & 91,01 & 2,24 & 98,16 & 233,28 \\
Mediana & 2,87 & 93,35 & 1,42 & 98,69 & 233,70 \\
CV & 13,34 & 7,38 & 92,05 & 2,10 & 1,68 \\
Curtose & 4,14 & 6,88 & 2,85 & 27,87 & 0,41 \\
Assimetria & $-1,90$ & $-2,36$ & 1,85 & $-4,81$ & $-0,68$ \\
$\mathrm{~d}$ & 0,01 & 0,01 & 0,01 & 0,01 & $0,15^{*}$ \\
\hline \multicolumn{5}{c}{$0,10-0,20 \mathrm{~m}$} \\
\hline Média & 2,51 & 86,54 & 3,64 & 230,80 \\
Mediana & 2,61 & 88,59 & 3,17 & 97,41 & 231,19 \\
CV & 16,76 & 8,90 & 62,71 & 2,27 & 1,55 \\
Curtose & 0,31 & 0,16 & 0,77 & 6,74 & 1,16 \\
Assimetria & $-0,74$ & $-0,90$ & 1,01 & $-2,23$ & 0,51 \\
$\mathrm{~d}$ & 0,02 & 0,01 & 0,01 & 0,01 & $0,15^{*}$ \\
\hline
\end{tabular}

${ }^{1} \mathrm{CV}$ : coeficiente de variação; ${ }^{2} \mathrm{~d}$ : teste de normalidade Kolmogorov-Smirnov, * significativo a $5 \%$ de probabilidade. 
Em busca da variação do atributo em relação à média, utilizou-se o coeficiente de variação $(\mathrm{CV})$, sendo tanto maior seu valor quanto maior a variação entre eles. Logo, a precisão de uma avaliação pode ser avaliada pelo CV e quanto menor, maior a homogeneidade dos dados. De acordo com a classificação de Warrick \& Nielsen (1980) os valores revelaram-se altos, médios e baixos. A camada de 0,05-0,10m demonstrou o maior e menor coeficiente de variação, para a classe de agregados de 2-1 mm, aquele que apresentou maior coeficiente de variação $(92,05)$ e o IEA, com menor coeficiente de variação $(0,36)$.

As classes de agregados 2-1 mm exibiram ocorrência de maior heterogeneidade no solo, concordando com resultados por Aquino et al. (2014) em estudos com variabilidade espacial em ambiente de TPA. As possíveis explicações para esse efeito, conforme relata Campos et al. (2011), é o elevado teor de matéria orgânica que se distribui de maneira descontínua na área em estudo, e ocasiona comportamentos diferenciados neste tipo de ambiente.

Os ajustes do semivariogramas experimentais e análise de dependência espacial são apresentados na Tabela 2. Todas as variáveis apresentaram dependência espacial, com exceção para as classes de agregados $>2 \mathrm{~mm}$ e 2-1 $\mathrm{mm}$ na profundidade $0,10-0,20 \mathrm{~m}$, os quais produziram efeito pepita puro. Este fato afirma a ausência da dependência espacial, considerando que a distância utilizada entre os pontos amostrados não detectou a dependência espacial para essas variáveis. Aquino et al. (2014) em área de terra preta arqueológica, encontrou, efeito pepita puro para classe de agregados $<1 \mathrm{~mm}$.

Os modelos esférico e exponencial foram os que melhor se ajustaram aos atributos. A MO e a classe de agregados 2-1,00 mm, ajustaram-se ao modelo esférico em todas as profundidades. Oliveira et al. (2013) em estudo com estabilidade de agregados e matéria orgânica de um Cambissolo Háplico sob cultivo de canade-açúcar, também ajustou esse modelo para essas variáveis. Neste sentido, Vieira et al. (2011) verificaram o modelo esférico para o DMG em um Latossolo cultivado em sistema de semeadura direta. Entretanto, o IEA nas profundidades 0,05-0,10 e 0,10-0,20 m, melhor se ajustaram ao modelo exponencial.

Observa-se que a continuidade espacial, vai diminuindo à medida que avança o perfil do solo, o que resulta na possibilidade de amostragens em intervalos menores na profundidade inferior, com o intuito de não perder a dependência espacial. Os atributos apresentaram diferentes valores de alcance, sendo a classe $>2,00 \mathrm{~mm}$ na profundidade 0,0-0,05 $\mathrm{m}$ apresentou maior alcance de 76m, enquanto que a classe 2-1 mm, com alcance de 12 $\mathrm{m}$. Os valores de alcance para os demais atributos foram entre 54 e $14 \mathrm{~m}$. É válido ressaltar, que o alcance é um importante parâmetro, pois é a distância limite da dependência espacial

A razão entre o efeito pepita e o patamar $(\mathrm{RD})$, se configura um indicativo da quantidade de variação ao acaso de um ponto para o outro ou o grau de dependência espacial. Dessa maneira, utilizou-se a classificação de Cambardella et al. (1994) para $\mathrm{RD}<25 \% ; 25 \%<\mathrm{RD}>75 \%$; e $\mathrm{RD}>75 \%$, para variabilidade forte, moderada e fraca, respectivamente. Portanto, os valores do DMG em todas as profundidades e classe de agregados 2-1 mm e o IEA nas profundidades 0,0-0,05 e 0,05-0,10m, caracterizaram por apresentar forte dependência espacial. Assim, estão relacionados com a variabilidade natural intrínseca do solo. Já as demais obtiveram dependência espacial moderada.

Os valores do coeficiente de determinação $\left(\mathrm{r}^{2}\right)$, revelam ótimo ajustes, com valores acima de 0,97 , com destaque para a MO e classe de agregados $>2,00 \mathrm{~mm}$. A validação cruzada (VC) oscilou entre 0,64 e 1,07. Já o DMG apresentou os menores valores de $\mathrm{C}_{0}$, indicando uma maior representatividade da variabilidade espacial desse atributo para área em estudo. 
Tabela 2 - Modelos e parâmetros estimados dos semivariogramas experimentais em relação ao diâmetro médio geométrico (DMG) e porcentagem dos agregados nas classes $>2 \mathrm{~mm}$ e $2-1 \mathrm{~mm}$, índice de estabilidade de agregados (IEA) e matéria orgânica (MO) em diferentes profundidades.

\begin{tabular}{|c|c|c|c|c|c|}
\hline \multirow[t]{2}{*}{ Parâmetros } & DMG & $>2 \mathrm{~mm}$ & $2-1 \mathrm{~mm}$ & IEA & $\mathrm{MO}$ \\
\hline & \multicolumn{5}{|c|}{$0-0,05 \mathrm{~m}$} \\
\hline Modelo & Esf. & Exp. & Esf. & Esf. & Esf. \\
\hline${ }^{1}\left(\mathrm{C}_{0}\right)$ & 0,01 & 6,20 & 0,02 & 0,65 & 40,80 \\
\hline${ }^{2}\left(\mathrm{C}_{0+} \mathrm{C}_{1}\right)$ & 0,07 & 12,41 & 0,90 & 1,31 & 90,45 \\
\hline${ }^{3} \mathrm{RD}$ & 14 & 50 & 2 & 50 & 45 \\
\hline${ }^{4} \mathrm{a}(\mathrm{m})$ & 20 & 76 & 15 & 35 & 47 \\
\hline$r^{2}$ & 0,61 & 0,95 & 0,55 & 0,75 & 0,89 \\
\hline \multirow[t]{2}{*}{${ }^{5} \mathrm{VC}$} & 0,72 & 0,85 & 0,82 & 0,64 & 1,07 \\
\hline & \multicolumn{5}{|c|}{$0,05-0,10 \mathrm{~m}$} \\
\hline Modelo & Exp. & Esf. & Esf. & Exp. & Esf. \\
\hline${ }^{1}\left(\mathrm{C}_{0}\right)$ & 0,01 & 0,75 & 0,07 & 0,009 & 3,61 \\
\hline${ }^{2}\left(\mathrm{C}_{0+} \mathrm{C}_{1}\right)$ & 0,10 & 17,59 & 2,05 & 0,10 & 12,23 \\
\hline${ }^{3} \mathrm{RD}$ & 10 & 4 & 3 & 9 & 29 \\
\hline${ }^{4} \mathrm{a}(\mathrm{m})$ & 19 & 14 & 12 & 26 & 23 \\
\hline$r^{2}$ & 0,81 & 0,50 & 0,50 & 0,86 & 0,97 \\
\hline \multirow[t]{2}{*}{${ }^{5} \mathrm{VC}$} & 0,72 & 0,82 & 0,97 & 0,86 & 0,67 \\
\hline & \multicolumn{5}{|c|}{$0,10-0,20 \mathrm{~m}$} \\
\hline Modelo & Exp. & - & - & Exp. & Esf. \\
\hline${ }^{1}\left(\mathrm{C}_{0}\right)$ & 0,01 & - & - & 0,06 & 6,78 \\
\hline${ }^{2}\left(\mathrm{C}_{0+} \mathrm{C}_{1}\right)$ & 0,15 & - & - & 0,55 & 13,57 \\
\hline${ }^{3} \mathrm{RD}$ & 6 & - & - & 11 & 49 \\
\hline${ }^{4} a(m)$ & 18 & - & - & 21 & 54 \\
\hline$r^{2}$ & 0,71 & - & & 0,71 & 0,92 \\
\hline${ }^{5} \mathrm{VC}$ & 1,00 & - & - & 0,75 & 0,86 \\
\hline
\end{tabular}

DMG: diâmetro médio geométrico; ${ }^{1}\left(\mathrm{C}_{0}\right)$ : efeito pepita; ${ }^{2}\left(\mathrm{C}_{0+} \mathrm{C}_{1}\right)$ : patamar; ${ }^{3} \mathrm{RD}$ : razão de dependência espacial; ${ }^{4} \mathrm{a}(\mathrm{m})$ : alcance; $\mathrm{r}^{2}$ : coeficiente de determinação; ${ }^{5} \mathrm{VC}$ : validação cruzada.

\section{ConClusões}

A porcentagem de agregados na classe $>2,00 \mathrm{~mm}$ apresentou valores acima de $80 \%$ indicando maior resistência à desagregação.

Os altos teores da Matéria Orgânica e estabilização dos agregados do solo foram observados na profundidade superficial

Houve o decréscimo nos valores das variáveis, DMG, IEA \%, classe de agregados $>2 \mathrm{~mm}(\%)$ e Matéria Orgânica em profundidade e, o inverso foi observado para a classe de agregados 2-1 mm (\%)

\section{REFERÊNCIAS}

Alho LC, Campos MCC, Silva DMP, Mantovanelli BC, Souza ZM. 2014. Variabilidade espacial da estabilidade de agregados e estoque de carbono em Cambissolo e Argissolo. Pesquisa Agropecuária Tropical, 44 (3) :246- 
254.

Aquino RE, Campos MCC, Oliveira IA, Marques Júnior J, Silva DMP, Silva DAP. 2014. Variabilidade espacial de atributos físicos de solos antropogênicos e não antropogênico na região de Manicoré, AM. Bioscence Journal, 30 (4): 988-997.

Araújo EA, Ker JC, Mendonça ES, Silva IR, Oliveira EK. 2011. Impacto da conversão floresta-pastagem nos estoques e na dinâmica do carbono e substâncias húmicas do solo no bioma Amazônico. Acta Amazônica, 41 (1) :103-114.

Brasil. 1978. Projeto Radam Brasil, folha SB.20, Purus. Ministério das Minas e Energia. Rio de Janeiro, Brasil. 561 p.

Campos MCC, Soares MDR, Aquino RE, Santos LAC, Mantovanelli BC. 2014. Distribuição espacial da resistência do solo à penetração e teor de água do solo em uma área de agrofloresta na região de Humaitá, AM. Comunicata Scientiae, 5 (4) :509-517.

Corrêa JC, Bull LT, Crusciol CAC, Moraes MH. 2009. Alteração de atributos físicos em Latossolo com aplicação superficial de escória de aciaria, lama cal, lodos de esgoto e calcário. Revista Brasileira de Ciência do Solo, 33 (3) :263- 272.

CPRM - Centro de Pesquisa de Recursos Minerais. Hidroclimatologia, geologia, recursos minerais, geomorfologia e unidades de paisagens. 2001. 93p. (Relatório Técnico).

Kemper WD e Chepil WS. 1965. Aggregate stability and size distribution. In: BLACK, C.A., ed. Methods of soil analysis. Madison, American Society of Agronomy, USA,510.

Luciano RV, Bertol I, Barbosa FT, Kurtz C, Fayad JA. 2010. Propriedades físicas e carbono orgânico do solo sob plantio direto comparados à mata natural, num Cambissolo Háplico. Revista de Ciências Agroveterinárias, 9 (1) : 09-19.

Neves CSVJ, Feller C, Kouakoua E. 2006. Efeito do manejo do solo e da matéria orgânica em água quente na estabilidade de agregados de um Latossolo Argiloso. Ciência Rural, 36 (5) :1410-1415.

Oliveira IA, Campos MCC, Aquino RE, Freitas L, Silva DMP. 2013. Spatial dependence of the aggregate stability and organic matter in a cambisol under sugar cane cultivation. Revista Caatinga, 26 (4) :1-9.

Oliveira IA, Campos MCC, Freitas L, Soares MDR. 2015. Caracterização de solos sob diferentes usos sob diferentes usos na região sul do Amazonas. Acta Amazonica, 45 (1) :1-12.

Raij BV, Quaggio JA, Cantarella J, Ferreira ME, LOPES AS, Bataglia O. 1987. Análise química do solo para fins de fertilidade. Campinas: Fundação Cargill,170p.

Santos LAC, Campos MCC, Aquino RE, Bergamim AC, Silva DMP, Masrques Júnior J, França ABC. 2013. Caracterização de terras pretas arqueológicas no Sul do Estado do Amazonas. Revista Brasileira de Ciência do solo, 37 (2) :825-836. 
Soares MDR, Campos, MCC, Oliveira IA, Cunha JM, Santos LAC, Fonseca JS, Souza ZMS. 2016. Atributos físicos do solo em áreas sob diferentes sistemas de usos na região de Manicoré, AM. Revista de Ciências Agrárias Amazonian Journal of Agricultural and Enviromental Sciencwa, 59 (1) : 9- 15.

Vasconcelos RFB, Cantalice JRB, Oliveira VS, Costa YDJ, Cavalcante DM. 2010. Estabilidade de agregados de um Latossolo Amarelo distrocoeso de Tabuleiro costeiro sob diferentes aportes de resíduos orgânicos da canade-açúcar. Revista Brasileira de Ciência do Solo, 34 (2) :309-316.

Vieira SR, Dechen SCF, Siqueira GM, Dufranc G. 2011. Variabilidade espacial de atributos físicos e químicos relacionados com o estado de agregação de dois Latossolos cultivados no sistema de semeadura direta. Bragantia, 70 (1):185-195.

Warrick AW e Nielsen DR. 1980. Spatial variability of soil physical properties in the field. In: HILLEL, D., ed. Applications of soil physics. New York, USA. p.344. 